\title{
DIFFERENTIAL ARCS AND REGULAR TYPES IN DIFFERENTIAL FIELDS
}

\author{
RAHIM MOOSA, ANAND PILLAY, AND THOMAS SCANLON
}

\begin{abstract}
We introduce differential arc spaces in analogy to the algebraic arc spaces and show that a differential variety in characteristic zero is determined by its arcs at a point. Using differential arcs, we show that if $\left(K,+, \times, \delta_{1}, \ldots, \delta_{n}\right)$ is a differentially closed field of characteristic zero with $n$ commuting derivations and $p \in S(K)$ is a regular type over $K$, then either $p$ is locally modular or there is a definable subgroup $G \leq(K,+)$ of the additive group having a regular generic type that is nonorthogonal to $p$.
\end{abstract}

\section{INTRODUCTION}

In many contexts, one may reduce the study of general partial differential equations to the study of linear PDEs. For example, when working with germs of meromorphic functions as coefficients and potential solutions, it is possible to construct from a general system of PDEs a corresponding system of linear PDEs whose solvability is equivalent to the solvability of the original system. However, this transformation requires an analytic reparametrization and does not make sense in the category of algebraic differential equations. Nevertheless, there is a technical sense in which the complexity of general algebraic partial differential equations is reducible to that of linear equations.

Recall that a (partial) differential field (with $n$ derivations) is a field $K$ given together with $n$ commuting derivations $\partial_{i}: K \rightarrow K$. (One could relax the commutation condition by requiring merely that the Lie algebra spanned by $\partial_{1}, \ldots, \partial_{n}$ is finite dimensional. Provided that one can solve enough differential equations on $K$, by a change of variables one may regard a differential field with initially noncommuting derivations as a differential field with commuting derivations.) By a system of (algebraic) partial differential equations over $K$ we mean a system of equations of the form $F_{1}\left(\left\{\partial_{1}^{\alpha_{1}} \cdots \partial_{n}^{\alpha_{n}}\left(x_{j}\right)\right\}\right)=0, \ldots, F_{\ell}\left(\left\{\partial_{1}^{\alpha_{1}} \cdots \partial_{n}^{\alpha_{n}}\left(x_{j}\right)\right\}\right)=0$ where $F_{1}, \ldots, F_{\ell} \in K\left[\left\{X_{j ; \alpha_{1}, \ldots, \alpha_{n}}\right\}_{1 \leq j \leq k ; \alpha \in \mathbb{N}^{n}}\right]$ are polynomials over $K$ in variables appropriate for coordinates $x_{1}, \ldots, x_{k}$ and their derivatives. A solution to a system of differential equations is given by a differential field extension $L \geq K$ and a point $a \in L^{k}$ for which all of these differential polynomials vanish when evaluated at $a$.

As with fields and ordinary algebraic equations, one can find differential fields in which every system of differential equations which could have a solution does: Speaking in the language of mathematical logic, in which we shall converse almost exclusively for the statement and proof of our main theorem, and specializing to characteristic zero, the theory of differential fields of characteristic zero with $n$

Date: 15 March 2007.

Pillay was partially supported by an NSF grant DMS-0300639.

Scanlon was partially supported by an Alfred P. Sloan Fellowship and NSF Grant DMS0301771. 
commuting derivations, $\mathrm{DF}_{0, n}$, has a model completion, $\mathrm{DCF}_{0, n}$, the theory of differentially closed fields of characteristic zero with $n$ commuting derivations. We regard the study of $\mathrm{DCF}_{0, n}$ as being synonomous with the study of algebraic differential equations.

Even for ordinary algebraic differential equations, it is not the case that every such differential equation is analyzable in terms of linear differential equations. For example, there are many differential equations whose solution sets are orthogonal to the solution sets of linear equations in the sense that if $X \subseteq K$ is the set of solutions to the equation in some differentially closed field $K$ and $V \subseteq K^{n}$ is a (finite dimensional over the constants) vector space of solutions to a system of linear differential equations, then for any $\Gamma \subseteq X \times V$ defined itself by differential equations, if both projections are (generically) surjective, then $\Gamma=X \times V$. Nevertheless, at least in the case of ordinary differential equations, such a situation is always explained by the geometric simplicity of $X$. What exactly we mean by this will be explained in a moment.

While it is not the case that every ordinary differential equation may be analyzed in terms of linear differential equations, they may be analyzed in terms of minimal equations (to be honest, it may be necessary to include infinitely many inequations as well). Here, we say that the subset $X \subseteq K$ of a differentially closed field defined by differential equations and inequations is minimal if it is infinite but for any polynomial $P\left(x_{0}, \ldots, x_{n}\right) \in K\left[x_{0}, \ldots, x_{n}\right]$ either every element $a$ of $X$ satisfies $P\left(a, \partial(a), \ldots, \partial^{n}(a)\right)=0$ or only finitely many do. Note that if $K$ is a differential field, then every differential equation on the constant field, $\mathcal{C}_{K}:=\{a \in K: \partial(a)=$ $0\}$, is simply a polynomial equation. Hence, $\mathcal{C}_{K}$ is minimal.

If $X$ and $Y$ are two minimal sets, then it is not hard to see that $X$ and $Y$ are nonorthogonal just in case there is a generically finite-to-finite correspondence $\Gamma \subseteq X \times Y$ defined by differential equations. It follows from this observation and the quantifier elimination theorem that nonorthogonality defines an equivalence relation on the class of minimal sets.

In theories of "finite rank" minimal sets play a fundamental role. We need a bit more notation to explain this: if $X$ and $Y$ are two definable sets we say that $X$ is internal to $Y$ if there is a definable map from some cartesian power of $Y$ onto $X$ (maybe definable with more parameters than those used to define $X$ and $Y$ ). For example the solution set $X$ to a linear ODE is a finite dimensional vector space over the constants $\mathcal{C}_{K}$ of the ambient differentially closed field $K$, and hence $X$ is internal to $\mathcal{C}_{K}$ : in fact it is in definable bijection with $\mathcal{C}_{K}^{n}$ after fixing a basis. Simplifying matters somewhat, if the ambient theory is stable, then for every definable set $X$ of finite rank there is a sequence of definable surjective maps and sets $X=X_{m} \rightarrow X_{m-1} \rightarrow \cdots \rightarrow X_{0}$, where $X_{0}$ is finite, such that each fibre of each map is internal to some minimal set. Thus the classification and structure of minimal sets in a given theory has great impact on the classification and structure of finite rank definable sets in that theory. Now although there are definable sets of infinite rank (dimension) in $\mathrm{DCF}_{0,1}$, such as affine 1-space over the ambient differentially closed field $K$, and there do exist nontrivial questions about infinite rank definable sets, the structure of finite rank definable sets is where most of the model-theoretic complexity lies in the case of $\mathrm{DCF}_{0,1}$.

A fundamental conjecture or conjectural dichotomy due to Boris Zilber says that any minimal set $X$ (or type) in a stable theory either has essentially a definable 
algebraically closed field structure or is geometrically very simple; we say locally modular. Local modularity of $X$ says more or less that there is no "rich" family of definable subsets of $X \times X$. The local modularity of all minimal sets in a theory has strong global consequences, for example that any finite rank definable group must be abelian-by-finite. Although this Zilber conjecture was shown to be false, it turns out to be true in many "natural" theories. In particular the dichotomy holds in $\mathrm{DCF}_{0,1}$ where it specialises to the statement that every nonlocally modular minimal set is nonorthogonal to the constants $\mathcal{C}_{K}$. This was proved by Hrushovski and Sokolović [5], and lies at the heart of the model-theoretic proof of the characteristic zero function field version of the Mordell-Lang conjecture [4]. The Hrushovski-Sokolović proof made crucial use of the central theorem on Zariski geometries of Hrushovski and Zilber [6]. Recently, Pillay and Ziegler [12] found a direct proof of this result (the Zilber conjecture for minimal types in $\mathrm{DCF}_{0,1}$ ) using "differential jet spaces" (higher dimension versions of differential tangent spaces). We should say that these methods and results also apply to minimal types of finite transcendence degree in $\mathrm{DCF}_{0, n}$ for $n>1$.

Everything we have said above applies only to finite rank definable sets (and types). However there are also infinite rank versions of minimal types. We call these regular types. In a superstable theory, arbitrary (possibly infinite rank) definable sets can be analysed as above but with minimal types replaced by regular types. So in an arbitrary superstable theory, the classification of regular types is fundamental. One can formulate the Zilber conjecture for regular types too (local modularity makes sense, see [11] for example). It is in general even more false than for minimal types, but in specific natural theories one may expect it to be true. In the case of $\mathrm{DCF}_{0,1}$ there is only one infinite rank regular type (up to nonorthogonality), namely the generic type of the ambient differentially closed field, for which Zilber's conjecture clearly holds. However in the case of PDE's, that is in the case of $\mathrm{DCF}_{0, n}$ for $n>1$ (which is superstable $[9,16]$ ), there will be many infinite rank regular types. It is worth saying at this point what regularity amounts to in $\mathrm{DCF}_{0, n}$ : The generic type of an irreducible differential variety $X$ will be regular if whenever $\left(Y_{a}: a \in Z\right)$ is a differential algebraic family of irreducible proper differential subvarieties of $X$, whose union is Kolchin dense in $X$, then any generic member $Y_{a}$ of the family is orthogonal to $X$.

The "natural" regular types in $\mathrm{DCF}_{0, n}$ come from the definable fields of constants: Suppose $\partial_{1}, \ldots, \partial_{n}$ are the distinguished commuting derivations, and $V$ is a $d$-dimensional subspace of the Lie algebra $\oplus_{i=1}^{n} K \partial_{i}$ spanned by these derivations, then the common constant field of $V, \mathcal{C}_{V}:=\{x \in K \mid \partial(x)=0$ for all $\partial \in V\}$, is a definable subfield of $K$ whose generic type is regular (of $U$-rank $\omega^{n-d}$ ). Zilber's conjecture for regular types in $\mathrm{DCF}_{0, n}$ has the concrete form: "any non-locally modular regular type is nonorthogonal to the generic type of such a definable field of constants". We have not succeeded in proving this conjecture. But what we have proved is that every non-locally modular regular type is nonorthogonal to a regular type which is the generic type of a definable subgroup of the additive group. As all such groups are defined by linear homogeneous differential equations, this gives some rigorous sense to the assertion that the geometric complexity of general algebraic differential equations is reducible to that of linear differential equations.

Our methods are heavily influenced by those of Pillay and Ziegler [12]. However when passing to our infinite-dimensional situation technical difficulties obstructed 
a smooth application of higher differential jet spaces. However, "differential arc spaces" work beautifully and we employ them to prove the above version of the dichotomy. In particular, we define the differential arc bundle $\mathcal{A}_{m}^{\Delta}(X)$ of a differential algebraic variety $X$. The fibre $\mathcal{A}_{m}^{\Delta}(X)_{a}$ at a sufficiently general point $a \in X$, while not itself a definable group, will be fibred by definable groups, in fact by differential tangent spaces. Using these differential arc spaces we prove that a regular non-locally modular type $p$ in $\mathrm{DCF}_{0, n}$ is nonorthogonal to a type realized in a suitable differential tangent space $G$. In the case of $\mathrm{DCF}_{0,1}$ with $p$ minimal, we recover the Hrushovski-Sokolović and Pillay-Ziegler results: $G$ will be finite dimensional over the constants, and so $p$ will be nonorthogonal to the constants.

Simplifying matters somewhat the proof of our main result on regular types in $\mathrm{DCF}_{0, n}$ essentially goes through the following steps:

(I) If $X$ is a differential algebraic variety defined over $k, a \in X$, and $c$ is some tuple such that $c$ is the canonical base of $\operatorname{tp}(a / k, c)$, then there is $m<\omega$ and tuple $d$ from $\mathcal{A}_{m}^{\Delta}(X)_{a}$ such that $c \in \operatorname{dcl}(k, a, d)$.

(II) If $p=\operatorname{tp}(a / k)$ is a non-locally modular regular type, then there is $b \in p^{e q}$ such that $\operatorname{tp}(b / k)$ has positive $p$-weight, and such that $p$ is nonorthogonal to $\operatorname{tp}(e / k, b)$ for some $e$ in the differential tangent space $T^{\Delta}(X)_{b}$ of the differential locus $X$ of $b$ at $b$.

(III) Show that in (II) $e$ can be chosen to be the regular generic of a definable subgroup of $T^{\Delta}(X)_{b}$.

Step (III) is somewhat involved, and depends on some additional data related to $\Delta$-types in the sense of Kolchin, as well as the structure of definable subgroups of powers of the additive group.

The authors would like to thank Eric Rosen as well as the referees for pointing out several inaccuracies in an earlier version of this paper.

\section{Differential arcs}

In this section we recall the construction of algebraic arc spaces and some of their properties, introduce differential arc spaces, and then demonstrate that differential varieties are determined by their arcs. Arc spaces were introduced by Nash to study resolution of singularities [10]. Kontsevich revived interest in arc spaces by using them as the basis for his theory of motivic integration and Denef and Loeser have systematically used these ideas [2]. The reader may wish to consult [8] for a discussion of the current state of research on arcs and their applications.

We recall the Weil trace construction, following [1]. If $\pi: T \rightarrow S$ is a map of schemes, then for any scheme $Y$ over $T$ we obtain a set-valued functor on the category of schemes over $S$ via $S^{\prime} \mapsto Y\left(S^{\prime} \times{ }_{S} T\right)$. By $Y\left(S^{\prime} \times{ }_{S} T\right)$ we mean the set of $\left(S^{\prime} \times{ }_{S} T\right)$-valued points of $Y$ over $T$; that is, the set of morphisms from $S^{\prime} \times{ }_{S} T$ to $Y$ over $T$. If this functor is representable, then the Weil restriction of $Y$ from $T$ to $S$, denoted $R_{T / S}(Y)$, is the representing object. That is, $R_{T / S}(Y)$ is a scheme over $S$ such that for any scheme $S^{\prime}$ over $S$, the $S^{\prime}$-valued points of $R_{T / S}(Y)$ over $S$ can be identified with the $\left(S^{\prime} \times{ }_{S} T\right)$-valued points of $Y$ over $T$. The Weil restriction exists under various hypotheses, the relevant ones for us are $T$ being finite over $S$ and $Y$ having the property that every finite set of points is contained in an affine open subset. 
Note that the hypothesis on a scheme that each finite set of points lies in an affine open subset follows from being a quasi-projective variety. As we will only need to consider affine varieties in our applications, we will implicitly assume this hypothesis whenever necessary.

We specialise to the case when $S$ is the spectrum of a field $k, T=\operatorname{Spec}\left(k^{(m)}\right)$ where $k^{(m)}:=k[\epsilon] /\left(\epsilon^{m+1}\right)$ for a natural number $m$, and $Y=X \otimes_{k} k^{(m)}$ for $X$ an algebraic variety over $k$. We view $k^{(m)}$ as a $k$-algebra under the natural map $a \mapsto a+0 \epsilon+\ldots+0 \epsilon^{m}$. The $m^{\text {th }}$ arc bundle of $X$ over $k$ is $R_{k^{(m)} / k}\left(X \otimes_{k} k^{(m)}\right)$, the Weil restriction of $X \otimes_{k} k^{(m)}$ from $\operatorname{Spec}\left(k^{(m)}\right)$ to $\operatorname{Spec}(k)$. We denote it by $\mathcal{A}_{m}(X / k)$, or just $\mathcal{A}_{m} X$ when there is no confusion. Note that $\mathcal{A}_{m} X$ is a scheme over $k$ (not necessarily reduced or irreducible).

For any $k$-algebra $R, \mathcal{A}_{m} X(R)$ can be identified with $X\left(R[\epsilon] /\left(\epsilon^{m+1}\right)\right)$. Indeed, by definition the $R$-points of $\mathcal{A}_{m} X$ correspond to the $\left(R \otimes_{k} k^{(m)}\right)$-points of $X \otimes_{k} k^{(m)}$ over $k^{(m)}$, and the latter are canonically the $R[\epsilon] /\left(\epsilon^{m+1}\right)$-points of $X$ over $k$.

In particular, $\mathcal{A}_{m} X(k)$ is identified with $X\left(k^{(m)}\right)$. So in the case that $X \subseteq \mathbb{A}^{\ell}$ is an affine variety we can write down the equations for $\mathcal{A}_{m} X \subseteq \mathbb{A}^{\ell(m+1)}$ as follows: If $X=\operatorname{Spec}\left(k\left[x_{1}, \ldots, x_{\ell}\right] /\left(\left\{f_{j}\right\}_{j \in J}\right)\right)$, then

$$
\mathcal{A}_{m} X=\operatorname{Spec}\left(k\left[\left\{x_{i, s}\right\}_{1 \leq i \leq \ell, 0 \leq s \leq m}\right] /\left(\left\{f_{j, t}\right\}_{j \in J, 0 \leq t \leq m}\right)\right.
$$

where $f_{j, t} \in k\left[\left\{x_{i, s}\right\}_{1 \leq i \leq \ell, 0 \leq s \leq m}\right]$ is defined by the identity

$$
f_{j}\left(\left(\sum_{t=0}^{m} x_{i, t} \epsilon^{t}\right)_{1 \leq i \leq \ell}\right)=\sum_{t=0}^{m} f_{j, t} \epsilon^{t}
$$

in the ring $k\left[\left\{x_{i, s}\right\}_{1 \leq i \leq \ell, 0 \leq s \leq m}, \epsilon\right] /\left(\epsilon^{m+1}\right)$.

If $f: X \rightarrow Y$ is a regular map of algebraic varieties over $k$, then $\mathcal{A}_{m}(f)$ : $\mathcal{A}_{m} X \rightarrow \mathcal{A}_{m} Y$ is the natural map which on $k$-points is given by $f$ evaluated on $X\left(k[\epsilon] /\left(\epsilon^{m+1}\right)\right)$. More explicitly, working locally assume that $X \subseteq \mathbb{A}^{\ell}, Y \subseteq \mathbb{A}^{r}$ and $f=\left(f_{1}, \ldots, f_{r}\right)$. Viewing $b \in \mathcal{A}_{m} X(k)$ as an element of $\mathbb{A}^{\ell}\left(k[\epsilon] /\left(\epsilon^{m+1}\right)\right)$ we have that $\mathcal{A}_{m}(f)(b)=\left(f_{1}(b), \ldots, f_{r}(b)\right)$ where the $f_{i}(b)$ are computed in the ring $k[\epsilon] /\left(\epsilon^{m+1}\right)$.

For $\ell \geq m$, the quotient map $k^{(\ell)} \rightarrow k^{(m)}$ corresponds to a natural transformation $\rho_{\ell, m}: \mathcal{A}_{\ell} \rightarrow \mathcal{A}_{m}$. Identifying $\mathcal{A}_{0}$ with the identity we write $\rho_{\ell, 0}$ as $\rho_{\ell}$. For $a \in X(k)$, the $\ell^{\text {th }}$ arc space $\mathcal{A}_{\ell} X_{a}$ of $X$ at $a$ is the fibre of $\rho_{\ell, X}: \mathcal{A}_{\ell} X \rightarrow X$ over $a$.

We recall some basic properties of algebraic arc spaces.

Lemma 2.1. Let $X$ be an algebraic variety over a field $k$ and $a \in X(k)$ a smooth point, then for any pair of natural numbers $\ell>m \geq 0$ the restriction of the map $\rho_{\ell, m}: \mathcal{A}_{\ell} X \rightarrow \mathcal{A}_{m} X$ to $\mathcal{A}_{\ell} X_{a}(k)$ is surjective onto $\mathcal{A}_{m} X_{a}(k)$.

Proof. This is essentially Hensel's Lemma. The problem is local, so we may and do assume that $X$ is affine. As $a$ is a smooth point, we may further assume that $X=V\left(g_{1}, \ldots, g_{r}\right) \subseteq \mathbb{A}^{d+r}$ where $d=\operatorname{dim}_{a} X$. Working by induction on $\ell$, it suffices to show that if $\widetilde{a} \in X\left(k[\epsilon] /\left(\epsilon^{m+1}\right)\right)$ is a lifting of $a$, then there is a point $\widehat{a} \in X\left(k[\epsilon] /\left(\epsilon^{m+2}\right)\right)$ lifting $\widetilde{a}$. Let $a^{\prime} \in \mathbb{A}^{d+r}\left(k[\epsilon] /\left(\epsilon^{m+2}\right)\right)$ be any lifting of $\widetilde{a}$. Letting $g:=\left(g_{1}, \ldots, g_{r}\right)$, note that $g\left(a^{\prime}\right) \bmod \left(\epsilon^{m+1}\right)=g(\widetilde{a})=0$ (working in the $\left.\operatorname{ring} k^{(m)}\right)$. So there is $b \in k^{r}$ such that $g\left(a^{\prime}\right)=b \epsilon^{m+1}$. To find $\widehat{a}$ one need only solve $d g_{a}(y)=-b$ in $k^{d+r}$ (which is possible since $d g_{a}$ has rank $r$ ), and set $\widehat{a}=a^{\prime}+y \epsilon^{m+1}$. Indeed, $g\left(a^{\prime}+y \epsilon^{m+1}\right)=g\left(a^{\prime}\right)+d g_{a}(y) \epsilon^{m+1}=0$, and so $\widehat{a} \in X\left(k[\epsilon] /\left(\epsilon^{m+2}\right)\right)$. 
The proof of Lemma 2.1 reveals the structure of the relative arc spaces as is made explicit in the following lemma. In particular, Lemma 2.2 will show that for $a \in X(k)$ smooth, $\mathcal{A}_{m} X_{a}$ is reduced and irreducible. In particular, if $X$ is a smooth irreducible variety then $\mathcal{A}_{m} X$ is an irreducible variety.

Lemma 2.2. Let $f: X \rightarrow Y$ be a regular map of algebraic varieties over the field $k$. Let $m$ be a natural number and $a_{m} \in \mathcal{A}_{m} X(k)$ such that $a:=\rho_{m}\left(a_{m}\right)$ is a smooth point of $X$ and $f(a)$ is a smooth point of $Y$. Let $\widetilde{X}$ be the fibre of $\rho_{m+1, m}$ : $\mathcal{A}_{m+1} X \rightarrow \mathcal{A}_{m} X$ over $a_{m}$, and $\tilde{Y}$ the fibre of $\rho_{m+1, m}: \mathcal{A}_{m+1} Y \rightarrow \mathcal{A}_{m} Y$ over $\mathcal{A}_{m}(f)\left(a_{m}\right)$. Then there are biregular maps $\psi_{X}: \widetilde{X} \rightarrow T_{a} X$ and $\psi_{Y}: \widetilde{Y} \rightarrow T_{f(a)} Y$ so that the following diagram is commutative

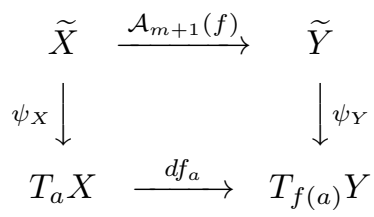

Proof. Working locally, we may write $X=V\left(g_{1}, \ldots, g_{r}\right) \subseteq \mathbb{A}^{t}$ and have $Y \subseteq$ $\mathbb{A}^{s}$. Since $\rho_{m}\left(a_{m}\right)=a \in X(k)$ is smooth, $a_{m} \in X\left(k[\epsilon] /\left(\epsilon^{m+1}\right)\right)$ has a lifting $a^{\prime} \in X\left(k[\epsilon] /\left(\epsilon^{m+2}\right)\right)$. If $b \in \mathbb{A}^{t}\left(k[\epsilon] /\left(\epsilon^{m+2}\right)\right)$ is another lifting, then we may write $b=a^{\prime}+y \epsilon^{m+1}$ for some $y \in k^{t}$. Let $g:=\left(g_{1}, \ldots, g_{r}\right)$. In order to have $b \in$ $X\left(k[\epsilon] /\left(\epsilon^{m+2}\right)\right)$ we need

$$
0=g(b)=g\left(a^{\prime}+\epsilon^{m+1} y\right)=g\left(a^{\prime}\right)+d g_{a}(y) \epsilon^{m+1}=d g_{a}(y) \epsilon^{m+1} .
$$

That is, $y \in T_{a} X$. Conversely, this equation shows that any such point in $T_{a} X$ gives rise to an element of $\widetilde{X}$. Likewise, we identify $\widetilde{Y}$ with $T_{f(a)} Y$. Writing an element of $\widetilde{X}(k)$ as $b=a^{\prime}+y \epsilon^{m+1}$, we see that

$$
\mathcal{A}_{m+1}(f)(b)=f\left(a^{\prime}+y \epsilon^{m+1}\right)=f\left(a^{\prime}\right)+d f_{a}(y) \epsilon^{m+1},
$$

which proves the claimed commutivity of the diagram.

As a consequence of Lemma 2.2, we see that arcs of dominant maps are themselves dominant. More precisely, we have the following lemma.

Lemma 2.3. Let $f: X \rightarrow Y$ be a dominant map of algebraic varieties over the field $k$. Suppose $a \in X(k)$ is smooth on $X, f(a) \in Y(k)$ is smooth on $Y$, and the rank of the differential of $f$ at a is equal to $\operatorname{dim} Y$. Then for every natural number $m$, the map $\mathcal{A}_{m}(f): \mathcal{A}_{m} X_{a}(k) \rightarrow \mathcal{A}_{m} Y_{f(a)}(k)$ is surjective.

Proof. We prove this lemma by induction on $m$ with the case of $m=0$ being trivial. In the case of $m+1$, let $y \in \mathcal{A}_{m+1} Y_{f(a)}(k)$. By induction, there is some $\bar{x} \in \mathcal{A}_{m} X_{a}(k)$ such that $\mathcal{A}_{m}(f)(\bar{x})=\rho_{m+1, m}(y)$. By Lemma 2.1 there is a point $\widetilde{x} \in \mathcal{A}_{m+1} X_{a}(k)$ with $\rho_{m+1, m}(\widetilde{x})=\bar{x}$. By Lemma 2.2 , we may identify $\rho_{m+1, m}^{-1}\{\bar{x}\}$ with $T_{a} X, \rho_{m+1, m}^{-1}\left\{\rho_{m+1, m}(y)\right\}$ with $T_{f(a)} Y$, and the restriction of $\mathcal{A}_{m+1}(f)$ to this fibre with $d f_{a}$. As such, the map is surjective between these fibres so that $y$ is in the range of $\mathcal{A}_{m+1}(f)$ as claimed.

Note that when the characteristic of $k$ is zero, the hypotheses of Lemma 2.3 hold for sufficiently general $a$ whenever $f$ is dominant. 
With the next lemma we note that subvarieties are determined by their arc spaces, at least in characteristic zero. We expect that this result has a straightforward characteristic-free algebraic proof and that it may even be well-known, but we could find no such proof in the literature. Our proof will make use of some model theory of algebraically closed valued fields, due essentially to Robinson [14]. Consider the following 3 -sorted language $L_{v a l}$ for valued fields: the field sort with the language of rings, the value group sort with the language of ordered groups, the residue field sort with the language of rings, and also the value map | - | from the field sort to the value group sort, and a 2-ary map Res from the field sort to the residue field sort, which takes $(x, y)$ to the residue of $x y^{-1}$ (and taking value 0 if $|x|>|y|$. Then in the language $L_{v a l}$, (i) the complete theory of an algebraically closed nontrivially valued field $(K, \Gamma, k)$ is determined by the pair $(\operatorname{char}(K), \operatorname{char}(k))$, and (ii) the theory of any algebraically closed nontrivially valued field has quantifier-elimination. We refer the reader to Theorem 2.1.1 of [3].

Lemma 2.4. Let $k$ be an algebraically closed field of characteristic zero and $X, Y \subseteq$ $Z$ irreducible algebraic varieties over $k$. If $a \in X(k) \cap Y(k)$ is a k-point on both $X$ and $Y$, then $X=Y$ if and only if $\mathcal{A}_{m} X_{a}(k)=\mathcal{A}_{m} Y_{a}(k)$ for all $m>0$.

Proof. Working locally, we may and do assume that $Z=\mathbb{A}^{m}$ is an affine space. Suppose that $X \neq Y$. Without loss of generality $X$ is not a subvariety of $Y$, hence there is $f \in I_{Y}, f \notin I_{X}$. The function field of $X, k(X)$, is the field of fractions of $k\left[x_{1}, \ldots, x_{m}\right] / I_{X}$. Extend the natural evaluation map $k\left[x_{1}, \ldots, x_{m}\right] / I_{X} \rightarrow k$ given by $\vec{x} \mapsto a$ to a $k$-place on $k(X)$ and let $v$ be the corresponding valuation. Let $w$ be an extension of $v$ to $L:=k(X)^{\text {alg }}$, the algebraic closure of $k(X)$. On the other hand, let $K=\bigcup_{\ell=1}^{\infty} k\left(\left(\epsilon^{\frac{1}{\ell}}\right)\right)$, where the union is naturally a direct limit. Let $w^{\prime}$ be the natural valuation on $K$ (with valuation ring $\bigcup_{\ell=1}^{\infty} k\left[\left[\epsilon^{\frac{1}{\ell}}\right]\right]$ and residue field $k$ ). As $k$ is algebraically closed of characteristic $0, K$ is algebraically closed. By points (i) and (ii) preceding the statement of this lemma, the valued fields $(L, w)$ and $\left(K, w^{\prime}\right)$ are elementarily equivalent over their common residue field $k$ (in the 3 -sorted language mentioned above). By construction, we have a point $b \in X\left(\mathcal{O}_{L, w}\right)$ for which $\operatorname{Res}(b, 1)=a$ and $f(b) \neq 0$. Indeed, $b$ is just $\vec{x} \bmod I_{X}$. Thus there is $c \in X\left(\mathcal{O}_{K, w^{\prime}}\right)$ with $\operatorname{Res}(c, 1)=a$ and such that $f(c) \neq 0$. For some $\ell$, $\left.c \in X\left(k\left[\epsilon^{\frac{1}{\ell}}\right]\right]\right) \backslash Y\left(k\left[\left[\epsilon^{\frac{1}{\ell}}\right]\right]\right)$. As $k[[\epsilon]] \cong_{k} k\left[\left[\epsilon^{\frac{1}{\ell}}\right]\right]$, we can find $\alpha \in X(k[[\epsilon]]) \backslash Y(k[[\epsilon]])$ which specialises to $a$. Considering the finite truncations of $\alpha$, it follows that for some $m$, there exists $d \in X\left(k[\epsilon] /\left(\epsilon^{m+1}\right)\right) \backslash Y\left(k[\epsilon] /\left(\epsilon^{m+1}\right)\right)$ which specialises to $a$. That is, $\mathcal{A}_{m} X_{a}(k) \neq \mathcal{A}_{m} Y_{a}(k)$. This proves the lemma.

By a $\Delta$-ring we will always mean a ring of characteristic zero equipped with $n$ commuting derivations $\Delta=\left\{\partial_{1}, \ldots, \partial_{n}\right\}$.

The arc space construction is very closely related to that of the prolongation spaces in differential algebraic geometry. Suppose $R$ is a $\Delta$-ring. Then the ring $R_{m}:=R\left[\eta_{1}, \ldots, \eta_{n}\right] /\left(\eta_{1}, \ldots, \eta_{n}\right)^{m+1}$ may be regarded an $R$-algebra via the "exponential" map $E: R \rightarrow R_{m}$ given by

$$
a \longmapsto \sum_{0 \leq \alpha_{1}+\cdots+\alpha_{n} \leq m} \frac{1}{\alpha_{1} ! \cdots \alpha_{n} !} \partial_{1}^{\alpha_{1}} \cdots \partial_{n}^{\alpha_{n}}(a) \eta_{1}^{\alpha_{1}} \cdots \eta_{n}^{\alpha_{n}}
$$

For $X$ an algebraic variety over a $\Delta$-field $k$ (or indeed any scheme over $k$ ), the $m^{\text {th }}$ prolongation $\tau_{m} X$ of $X$ is the Weil restriction of $X \otimes_{E} k_{m}$ from $\operatorname{Spec}\left(k_{m}\right)$ to $\operatorname{Spec}(k)$. That is, $\tau_{m} X=R_{k_{m} / k}\left(X \otimes_{E} k_{m}\right)$. Note that we are taking the base 
change $X \otimes_{E} k_{m}$ with respect to the exponential map while we are taking the Weil restriction with respect to the standard $k$-algebra structure on $k_{m}$ given by the inclusion $k \rightarrow k_{m}$. So when $n=1$ and $\partial_{1}=0, \tau_{m}$ and $\mathcal{A}_{m}$ agree.

From the reduction (or quotient) maps $k_{\ell} \rightarrow k_{m}$ (for $\ell \geq m$ ) we see that the prolongations form a projective system $\pi_{\ell, m}: \tau_{\ell} \rightarrow \tau_{m}$. Identifying $\tau_{0}$ with the identity, we write $\pi_{\ell, 0}$ as $\pi_{\ell}$ and have a map $\pi_{\ell}: \tau_{\ell} X \rightarrow X$. The map on $k$-points given by $x \longmapsto \sum_{0 \leq \alpha_{1}+\cdots+\alpha_{n} \leq m} \frac{1}{\alpha_{1} ! \cdots \alpha_{n} !} \partial_{1}^{\alpha_{1}} \cdots \partial_{n}^{\alpha_{n}}(x) \eta_{1}^{\alpha_{1}} \cdots \eta_{n}^{\alpha_{n}}$ gives a section to $\pi_{m}$, which we denote by $\nabla_{m}: X(k) \rightarrow \tau_{m} X(k)$.

The connections between the arc and prolongation spaces run deeper than merely the fact that the arc spaces may be regarded as prolongation spaces relative to a trivial derivation. Indeed, an analogue of Lemma 2.2 holds for prolongation spaces. That is, the fibres of the $(m+1)^{\text {st }}$ prolongation space over the $m^{\text {th }}$ prolongation space are biregularly isomorphic with torsors of cartesian powers of the tangent space of the base. For the sake of completeness, we state this result precisely. Before doing so, let us fix some notation. For a pair of natural numbers $(m, n)$, let $p_{m, n}:=\#\left\{\left(\ell_{1}, \ldots, \ell_{n}\right) \in \mathbb{N}^{n}: \sum_{i=1}^{n} \ell_{i}=m\right\}$ be the number of partitions of $m$ into $n$ parts. For $N$ a natural number and $f: X \rightarrow Y$ a map of varieties, we write $X^{\times_{Y} N}$ for the $N^{\text {th }}$ cartesian power of $X$ over $Y$.

Lemma 2.5. Suppose $X$ is an algebraic variety over a $\Delta$-field $k$ and $m$ is a positive integer. There is a functorial action

$$
\mu_{X}:\left((T X)^{\times{ }_{X} p_{m+1, n}} \times_{X} \tau_{m} X\right) \times_{\tau_{m} X} \tau_{m+1} X \rightarrow \tau_{m+1} X
$$

over $\tau_{m} X$. Moreover, for any point $a \in \tau_{m} X(k)$ if the fibre of $\tau_{m+1} X \rightarrow \tau_{m} X$ over $a$ is nonempty, then this action makes that fibre into a principal homogeneous space for $\left(T_{\pi_{m}(a)} X\right)^{p_{m+1, n}}$. In particular, if $X$ is a smooth, irreducible variety then $\tau_{m+1} X$ is a smooth, irreducible (reduced) variety.

Proof. We describe the action of $(T X)^{\times}{ }^{p_{m+1, n}}$ on points of $\tau_{m+1} X$ in terms of the functor represented by the Weil trace construction which identifies $\tau_{m+1} X(R)$ with $\left(X \otimes_{E} k_{m+1}\right)\left(R_{m+1}\right)$. It suffices to consider the case that $X$ is affine. Suppose that $X=\operatorname{Spec}\left(k\left[z_{1}, \ldots, z_{r}\right] /\left(g_{1}, \ldots, g_{s}\right)\right)$. Fix a $k$-algebra $R$, a point $c \in X(R)$, a sequence of points $v_{\alpha} \in T_{c} X(R)$ of the tangent space indexed by $\alpha \in \mathbb{N}^{n}$ with $\sum_{i=1}^{n} \alpha_{i}=m$, and a point $b \in\left(X \otimes_{E} k_{m+1}\right)\left(R_{m+1}\right)$ which reduces to $c$ under the reduction map. Then the proposed action is given by $b \mapsto b+\sum v_{\alpha} \eta^{\alpha}$. Indeed,

$$
\begin{aligned}
g_{i}^{E}\left(b+\sum v_{\alpha} \eta^{\alpha}\right) & =g_{i}^{E}(b)+\sum_{\alpha}\left(d g_{i}^{E}\right)_{c}\left(v_{\alpha}\right) \eta^{\alpha} \\
& =g_{i}^{E}(b)+\sum_{\alpha}\left(d g_{i}\right)_{c}\left(v_{\alpha}\right) \eta^{\alpha} \\
& =0
\end{aligned}
$$

as $g_{i}^{E}(b)=0$ (since $\left.b \in\left(X \otimes_{E} k_{m+1}\right)\left(R_{m+1}\right)\right)$ and $\left(d g_{i}\right)_{c}\left(v_{\alpha}\right)=0$ (since $v_{\alpha} \in$ $\left.T_{c} X(R)\right)$. Since $b$ and $b+\sum v_{\alpha} \eta^{\alpha}$ reduce to the same point in $\left(X \otimes_{E} k_{m}\right)\left(R_{m}\right)$, this action is over $\tau_{m} X$.

The same computations establish functoriality and show that the difference of two points in $\left(X \otimes_{E} k_{m+1}\right)\left(R_{m+1}\right)$ with the same image in $\left(X \otimes_{E} k_{m}\right)\left(R_{m}\right)$ has the form $\sum v_{\alpha} \eta^{\alpha}$ where each $v_{\alpha}$ belongs to $T_{c} X(R)$. 
Remark 2.6. We have already pointed out that in general the arc bundles and prolongation bundles need not be reduced. This will not pose a problem for us for the following two reasons: 1) we will mostly be considering arc and prolongation spaces at smooth points where they are reduced and irreducible, and 2) we will be working with the $k$-rational points of these spaces, where $k$ is a field, and such points depend only on the underlying reduced variety.

Lemma 2.7. $\tau_{m} \mathcal{A}_{r}(X)=\mathcal{A}_{r} \tau_{m}(X)$.

Proof. Let $k^{(r)}:=k[\epsilon] /(\epsilon)^{r+1}$ viewed as a $k$-algebra via the inclusion $k \rightarrow k^{(r)}$. Let $K_{s}:=k^{(r)} \otimes_{s} k_{m}$ (here $s$ stands for standard) and $K_{E}:=k^{(r)} \otimes_{E} k_{m}$. We have the following two commuting squares:
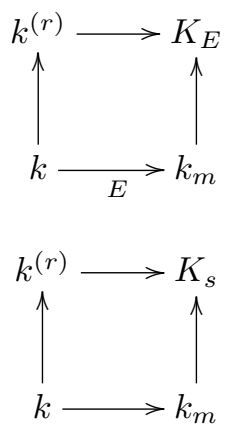

Now, given any scheme $U$ over $k$

$$
\begin{aligned}
\tau_{m} \mathcal{A}_{r}(X)(U) & =\operatorname{Hom}_{k_{m}}\left(U \otimes_{k} k_{m}, R_{k^{(r)} / k}\left(X \otimes_{k} k^{(r)}\right) \otimes_{E} k_{m}\right) \\
& =\operatorname{Hom}_{k_{m}}\left(U \otimes_{k} k_{m}, R_{K_{E} / k_{m}}\left(X \otimes_{k} k^{(r)} \otimes_{k^{(r)}} K_{E}\right)\right) \\
& =\operatorname{Hom}_{K_{E}}\left(U \otimes_{k} k_{m} \otimes_{k_{m}} K_{E}, X \otimes_{k} k^{(r)} \otimes_{k^{(r)}} K_{E}\right) \\
& =\operatorname{Hom}_{K_{E}}\left(U \otimes_{k} k_{m} \otimes_{k_{m}} K_{E}, X \otimes_{E} k_{m} \otimes_{k_{m}} K_{E}\right)
\end{aligned}
$$

where the second equality is by the fact that Weil restrictions are compatible with base change, and the final equality uses the first commuting square above. On the other hand

$$
\begin{aligned}
\mathcal{A}_{r} \tau_{m}(X)(U) & =\operatorname{Hom}_{k^{(r)}}\left(U \otimes_{k} k^{(r)}, R_{k_{m} / k}\left(X \otimes_{E} k_{m}\right) \otimes_{k} k^{(r)}\right) \\
& =\operatorname{Hom}_{k^{(r)}}\left(U \otimes_{k} k^{(r)}, R_{K_{s} / k^{(r)}}\left(X \otimes_{E} k_{m} \otimes_{k_{m}} K_{s}\right)\right) \\
& =\operatorname{Hom}_{K_{s}}\left(U \otimes_{k} k^{(r)} \otimes_{k^{(r)}} K_{s}, X \otimes_{E} k_{m} \otimes_{k_{m}} K_{s}\right) \\
& =\operatorname{Hom}_{K_{s}}\left(U \otimes_{k} k_{m} \otimes_{k_{m}} K_{s}, X \otimes_{E} k_{m} \otimes_{k_{m}} K_{s}\right)
\end{aligned}
$$

where again the second equality is by the fact that Weil restrictions are compatible with base change and the last equality uses the second commuting square above. The lemma now follows once we observe that $K_{E}$ and $K_{s}$ are canonically isomorphic over $k_{m}$ (they differ only as $k$-algebras).

It is sometimes convenient to view the higher prolongations as canonically embedded in the iterated prolongations. That is, instead of $\tau_{m} X$, one might consider $\tau^{m} X:=\overbrace{\tau \circ \cdots \circ \tau}^{m \text { times }} X$. The corresponding section $\nabla^{m}: X(k) \rightarrow \tau^{m} X(k)$ 
is given by $\nabla^{m}:=\overbrace{\nabla \circ \cdots \circ \nabla}^{m \text { times }}$. The map from $k\left[\eta_{1}, \ldots, \eta_{n}\right] /\left(\eta_{1}, \ldots, \eta_{n}\right)^{m+1}$ to $k\left[\xi_{1,1}, \ldots, \xi_{1, m} ; \ldots ; \xi_{n, 1}, \ldots, \xi_{n, m}\right] /\left(\left(\left\{\xi_{i, j}\right\}_{1 \leq i \leq n}\right)_{1 \leq j \leq m}^{2}\right)$ given by $\eta_{i} \mapsto \sum_{j} \xi_{i, j}$ induces an embedding $\tau_{m} X \hookrightarrow \tau^{m} X$. Under this embedding, the map $\pi_{\ell, m}: \tau_{\ell} X \rightarrow$ $\tau_{m} X$ (for $\ell \geq m$ ) extends to the map $\overbrace{\pi_{1} \circ \cdots \circ \pi_{1}}^{\ell-m \text { times }}: \tau^{\ell} X \rightarrow \tau^{m} X$, which we will also denote by $\pi_{\ell, m}$.

Differentially (or $\Delta$-) closed fields (of characteristic zero) may be characterized as algebraically closed $\Delta$-fields $K$ for which given a projective system of dominant maps of irreducible algebraic varieties over $K,\left\langle\mu_{\ell, m}: X_{\ell} \rightarrow X_{m}\right\rangle$, for which $X_{\ell}$ is a closed subvariety of $\tau^{\ell-m} X_{m}$ and $\mu_{\ell, m}$ is the restriction of $\pi_{\ell-m}$ to $X_{\ell}$, there is a point $a \in X_{0}(K)$ such that $\nabla^{\ell}(a) \in X_{\ell}(K)$ for all $\ell$.

Let us fix a sufficiently saturated differentially closed field $k$, which we treat as a universal domain for differential algebraic geometry. We will treat $\Delta$-varieties $X$ as $\Delta$-closed subsets of $\bar{X}(k)$, where $\bar{X}$ is the Zariski closure of $X$. That is, we do not take a scheme-theoretic approach to $\Delta$-varieties.

Given an irreducible $\Delta$-variety $X$, and a natural number $\ell$, consider the irreducible algebraic variety

$$
X_{\ell}:=\text { the Zariski closure of } \nabla_{\ell}(X) \text { in } \tau_{\ell} \bar{X}(k) .
$$

Then $X$ is determined, as a $\Delta$-closed subset of $\bar{X}$, by its prolongation sequence $\left\langle\pi_{\ell, m}: X_{\ell} \rightarrow X_{m} \mid \ell \geq m\right\rangle$. Indeed $X=\left\{a \in \bar{X}(k): \nabla_{\ell}(a) \in X_{\ell}(k)\right.$, for all $\left.\ell \geq 0\right\}$. Conversely, suppose $\left\langle X_{\ell} \subseteq \tau_{\ell} Y \mid \ell \geq 0\right\rangle$ is a sequence of irreducible algebraic subvarieties of an algebraic variety $Y$ such that:

(a) $\pi_{\ell+1, \ell}$ restricts to a dominant map from $X_{\ell+1}$ to $X_{\ell}$, and

(b) after embedding $\tau_{\ell} Y$ in $\tau^{\ell} Y$ and $\tau_{\ell+1} Y$ in $\tau^{\ell+1} Y, X_{\ell+1}$ is a closed subvariety of $\tau X_{\ell}$,

then there exists a (unique) $\Delta$-subvariety $X$ of $Y$ such that $\tau_{\ell} X=X_{\ell}$.

In the remainder of this section, we work towards a differential analogue of arc spaces for which Lemma 2.4 will hold true. Given a $\Delta$-variety $X$ over $k$, one could mimic the Weil trace construction in the category of $\Delta$-schemes over $k$, and define the $m^{\text {th }}(\Delta$-)arc bundle of $X$ to be the object which represents the functor $T \mapsto X \otimes_{k} k^{(m)}\left(T \otimes_{k} k^{(m)}\right)$, where $k^{(m)}$ is made into a $\Delta$-ring by taking $\epsilon$ to be $\Delta$-constant. To really make this work one would need to use a good theory of $\Delta$-schemes. We proceed differently however. Our approach is to assume that $X$ is given to us as a $\Delta$-closed subset of an algebraic variety $\bar{X}$, and then to define $\mathcal{A}_{m}^{\Delta} X$ by defining its prolongation sequence:

Definition 2.8. Suppose $X \subseteq \bar{X}(k)$ is an irreducible $\Delta$-subvariety of the algebraic variety $\bar{X}$ over $k$, and $m$ is a natural number. For each natural number $\ell$ let $Z_{\ell}$ be the unique irreducible component of $\left(\mathcal{A}_{m}\left(X_{\ell}\right)\right)_{\text {red }}$ that projects dominantly to $X_{\ell}$. ${ }^{1}$ So $Z_{\ell}$ is an irreducible algebraic subvariety of $\mathcal{A}_{m} \tau_{\ell} \bar{X}$. Identifying the latter with $\tau_{\ell} \mathcal{A}_{m} \bar{X}$ by Lemma 2.7 , we view $Z_{\ell}$ as an irreducible algebraic subvariety of $\tau_{\ell} \mathcal{A}_{m} \bar{X}$. We define the $m^{\text {th }}$ differential arc bundle of $X$ as

$$
\mathcal{A}_{m}^{\Delta} X:=\left\{a \in \mathcal{A}_{m} \bar{X}(k): \nabla_{\ell}(a) \in Z_{\ell}(k) \text { for all } \ell \geq 0\right\} .
$$

\footnotetext{
${ }^{1}$ Note that such a component exists since the generic fibres of $\mathcal{A}_{m} X_{\ell}$ over $X_{\ell}$ are reduced and irreducible. Moreover, if $X_{\ell}$ is smooth then $Z_{\ell}=\mathcal{A}_{m}\left(X_{\ell}\right)$.
} 
For $a \in X$, we define the $m^{\text {th }}$ arc space of $X$ at $a$ to be $\mathcal{A}_{m}^{\Delta} X_{a}$, the fibre above $a$ of the map $\rho_{m}: \mathcal{A}_{m} \bar{X} \rightarrow \bar{X}$ restricted to $\mathcal{A}_{m}^{\Delta} X$.

Proposition 2.9. Suppose $X \subseteq \bar{X}(k)$ is an irreducible $\Delta$-subvariety of the algebraic variety $\bar{X}$ over $k$, and $m$ is a natural number. Let $Z_{\ell} \subseteq \tau_{\ell} \mathcal{A}_{m} \bar{X}$ be as in Definition 2.8. The natural maps $\tau_{s} \mathcal{A}_{m} \bar{X} \rightarrow \tau_{t} \mathcal{A}_{m} \bar{X}$ for $s \geq t$ restrict to produce $a$ prolongation sequence $\left\langle Z_{s} \rightarrow Z_{t} \mid s \geq t\right\rangle$. Hence, $\mathcal{A}_{m}^{\Delta} X$ is a $\Delta$-subvariety of $\mathcal{A}_{m} \bar{X}$ having this sequence as it's prolongation sequence.

Moreover, if $a \in X$ is smooth in that $\nabla_{s}(a)$ is a smooth point on $X_{s}$ for each $s$, and $d\left(\pi_{s, t}\right)_{\nabla_{s}(a)}$ has full rank for every $s \geq t$, then $\overline{\nabla_{s}\left(\mathcal{A}_{m}^{\Delta} X_{a}\right)}=\mathcal{A}_{m}\left(X_{s}\right)_{\nabla_{s}(a)}$.

Proof. Viewing the higher prolongations of $\bar{X}$ as embedded in its iterated prolongations, we have that $X_{s+1} \subseteq \tau\left(X_{s}\right)$ for every $s$. As $\mathcal{A}_{m}$ preserves inclusions we have $\mathcal{A}_{m}\left(X_{s+1}\right) \subseteq \mathcal{A}_{m} \circ \tau\left(X_{s}\right)=\tau \mathcal{A}_{m}\left(X_{s}\right)$. It follows that $Z_{s+1} \subseteq \tau\left(Z_{s}\right)$. Also, the maps $\pi_{s, t}: X_{s} \rightarrow X_{t}$ are dominant, and so by Lemma 2.3 the maps $\mathcal{A}_{m}\left(\pi_{s, t}\right): \mathcal{A}_{m}\left(X_{s}\right) \rightarrow \mathcal{A}_{m}\left(X_{t}\right)$ are dominant. Hence $Z_{s} \rightarrow Z_{t}$ are dominant. So $\left\langle Z_{s} \rightarrow Z_{t} \mid s \geq t\right\rangle$ is a prolongation sequence, and from the definition of $\mathcal{A}_{m}^{\Delta} X$ we see that it is the prolongation sequence of this $\Delta$-variety.

For the "moreover" clause, note that by the smoothness of $\nabla_{s}(a), \mathcal{A}_{m}\left(X_{s}\right)_{\nabla_{s}(a)}$ is reduced and irreducible, and that $\mathcal{A}_{m}\left(X_{s}\right)_{\nabla_{s}(a)}=\left(Z_{s}\right)_{\nabla_{s}(a)}$. Hence

$$
\mathcal{A}_{m}^{\Delta} X_{a}=\left\{b \in \mathcal{A}_{m} \bar{X}_{a}(k): \nabla_{s}(b) \in \mathcal{A}_{m}\left(X_{s}\right)_{\nabla_{s}(a)}(k) \text { for all } s \geq 0\right\} .
$$

The smoothness of $a$ also ensures that $\left\langle\mathcal{A}_{m}\left(X_{s}\right)_{\nabla_{s}(a)} \rightarrow \mathcal{A}_{m}\left(X_{t}\right)_{\nabla_{t}(a)} \mid s \geq t\right\rangle$ is a prolongation sequence. It follows that this sequence is the prolongation sequence of $\mathcal{A}_{m}^{\Delta} X_{a}$, and so $\overline{\nabla_{s}\left(\mathcal{A}_{m}^{\Delta} X_{a}\right)}=\mathcal{A}_{m}\left(X_{s}\right)_{\nabla_{s}(a)}$, as desired.

It should be noted that if $X$ is an algebraic variety over $k$ and $a \in X(k)$ is a smooth point, then the $\Delta$-arc space and algebraic arc space agree: or more precisely, $\mathcal{A}_{m}^{\Delta} X_{a}=\mathcal{A}_{m} X_{a}(k)$.

From Proposition 2.9 we may derive several useful corollaries.

Lemma 2.10. Suppose $X$ and $Y$ are irreducible $\Delta$-subvarieties of an algebraic variety over $k$, and $a \in X \cap Y$ is a common smooth point (in the sense of Proposition 2.9). Then $X=Y$ if and only if $\mathcal{A}_{m}^{\Delta} X_{a}=\mathcal{A}_{m}^{\Delta} Y_{a}$ for all $m>0$.

Proof. If $\mathcal{A}_{m}^{\Delta} X_{a}=\mathcal{A}_{m}^{\Delta} Y_{a}$, then $\overline{\nabla_{s}\left(\mathcal{A}_{m}^{\Delta} X_{a}\right)}=\overline{\nabla_{s}\left(\mathcal{A}_{m}^{\Delta} Y_{a}\right)}$, and so by the "moreover" clause of Proposition 2.9, $\mathcal{A}_{m}\left(X_{s}\right)_{\nabla_{s}(a)}(k)=\mathcal{A}_{m}\left(Y_{s}\right)_{\nabla_{s}(a)}(k)$ for all $s \geq 0$. Fixing $s$, the above equality for all $m$ implies by Lemma 2.4 that $X_{s}=Y_{s}$. Hence $X$ and $Y$ have the same prolongation sequence, and are therefore equal.

The $\Delta$-tangent bundle $T^{\Delta} X$ of a $\Delta$-variety $X$ was introduced by Kolchin. Explicit equations for this space can be found in section VIII.2 of [7]. The following lemma shows that we recover the $\Delta$-tangent spaces as the first $\Delta$-arc spaces.

Lemma 2.11. Let $X \subseteq \mathbb{A}^{\ell}$ be a $\Delta$-subvariety of affine space. For $a \in X$ smooth in the sense of Proposition 2.9, $\mathcal{A}_{1}^{\Delta} X_{a}$ is canonically isomorphic to $T^{\Delta} X_{a}$.

Moreover, the map given by Lemma 2.2 which identifies the fibres of $\mathcal{A}_{m+1} \bar{X}_{a} \rightarrow$ $\mathcal{A}_{m} \bar{X}_{a}$ with $T_{a} \bar{X}$ restricts to an isomorphism of the fibres of $\mathcal{A}_{m+1}^{\Delta} X_{a} \rightarrow \mathcal{A}_{m}^{\Delta} X_{a}$ with $T_{a}^{\Delta} X$.

Proof. We can and do systematically identify the first arc bundle of an algebraic variety with its tangent bundle. 
Suppose $a \in X$ is smooth and $b \in T_{a} \bar{X}(k)$. By definition, $b \in \mathcal{A}_{1}^{\Delta} X$ if and only if $\nabla_{r}(a, b) \in T\left(X_{r}\right)(k)$ for all $r \geq 0$, where $T\left(X_{r}\right)$ is viewed as an algebraic subvariety of $\tau_{r}(T \bar{X})$ under the identification of $T\left(\tau_{r} \bar{X}\right)$ with $\tau_{r}(T \bar{X})$. Note that under this last identification, $\nabla_{r}(a, b)$ becomes $\left(\nabla_{r} a, \nabla_{r} b\right)$. That is, $(a, b) \in \mathcal{A}_{1}^{\Delta} X$ if and only if for all $r \geq 0$

$$
\sum_{1 \leq i \leq n^{r} \ell(r+1)} \frac{\partial P}{\partial x_{i}}\left(\nabla_{r} a\right) \cdot\left[\nabla_{r} b\right]_{i}=0
$$

for all $P$ in the defining ideal of $X_{r}$, where $\left[\nabla_{r} b\right]_{i}$ denotes the $i$ th coordinate of $\nabla_{r} b$. These are exactly the equations given by Kolchin in section VIII.2 of [7] for the $\Delta$-tangent bundle.

The "moreover" clause now follows by inspecting the map given in Lemma 2.2. In particular, if $c \in \mathcal{A}_{m}^{\Delta} X_{a}$ and $r \geq 0$, then by Proposition 2.9, $\nabla_{r} c \in \mathcal{A}_{m}\left(X_{r}\right)_{\nabla_{r} a}$ (which is reduced and irreducible by the smoothness of $\nabla_{r} a$ ), and the following diagram commutes:

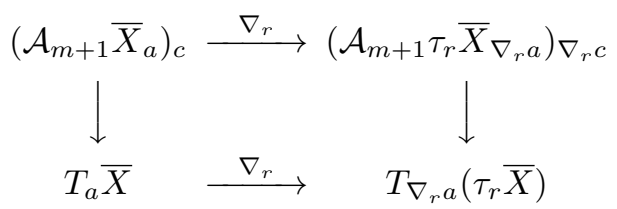

where the vertical arrows are the maps given by Lemma 2.2 (applied to $\bar{X}$ and $\tau_{r} \bar{X}$ respectively). It follows that $\left(\mathcal{A}_{m+1}^{\Delta} X_{a}\right)_{c}$ is identified with $T_{a}^{\Delta} X$, as desired.

Finally in this section, let us recall Kolchin's notions of $\Delta$-type and typical $\Delta$-dimension. This material is from section 0.3 of [7]. If $a$ is some finite tuple from $k$, and $F$ is a $\Delta$-subfield of $k$, then there is a polynomial $K_{a / F}(y)$ such that for sufficiently large natural numbers $r, K_{a / F}(r)$ is the transcendence degree of $F\left(\nabla_{r}(a)\right)$ over $F$. (Note that if $a$ is an $m$-tuple then $\nabla_{r}(a)$ is a $k$-rational point of $\tau_{r} \mathbb{A}^{m}$ and hence is itself a finite tuple from $k$ - so the transcendence degree of $F\left(\nabla_{r}(a)\right)$ over $F$ makes sense.) It bears noting that the coefficients of the Kolchin polynomial are merely rational numbers and not necessarily integers. However, the leading coefficient must be positive and

$$
\{(\operatorname{deg}(P) \text {, leading coefficient }(P)) \mid P \text { a Kolchin polynomial }\}
$$

is well-ordered as a subset of $\mathbb{N} \times \mathbb{Q}$. The latter follows from the fact that the set of Kolchin polynomials is well-ordered under eventually domination (see [15]).

The degree of $K_{a / F}$ is called the $\Delta$-type of $a$ over $F$ and the leading coefficient of $K_{a / F}$ is called the typical $\Delta$-dimension of $a$ over $F$, which we write here as $\operatorname{dim}_{\Delta}(a / F)$. Note that $\Delta$-type zero corresponds to the $\Delta$-subfield generated by $a$ over $F$ having finite transcendence degree over $F$. These two quantities are $\Delta$ birational invariants of $a$ over $F$, namely if $b$ generates over $F$ the same $\Delta$-field as $a$, then $a$ and $b$ have the same $\Delta$-type and typical $\Delta$-dimension over $F$.

If $X$ is an $F$-irreducible $\Delta$-variety, then define the $\Delta$-type and typical $\Delta$-dimension of $X$ to be those of $a$ over $F$ where $a$ is a generic point of $X$ over $F$. Likewise we define the Kolchin polynomial $K_{X}$ of $X$ to be $K_{a / F}$. Note that the transcendence degree of $F\left(\nabla_{r}(a)\right)$ over $F$ is the the dimension of the variety $X_{r}$. Hence if $Y \subseteq X$ is a $\Delta$-subvariety with the same Kolchin polynomial then $Y=X$.

Corollary 2.12. Let $X \subseteq \mathbb{A}^{\ell}$ be a $\Delta$-subvariety of affine space, $m$ a positive integer, and $a \in X$ a smooth point in the sense of Proposition 2.9. Then $K_{\mathcal{A}_{m}^{\Delta} X_{a}}=m K_{X}$. 
Proof. By the "moreover" clause of Proposition 2.9, $\overline{\nabla_{r}\left(\mathcal{A}_{m}^{\Delta} X_{a}\right)}=\mathcal{A}_{m}\left(X_{r}\right)_{\nabla_{r}(a)}$. But since $\nabla_{r}(a)$ is a smooth point of the algebraic variety $X_{r}, \operatorname{dim} \mathcal{A}_{m}\left(X_{r}\right)_{\nabla_{r}(a)}=$ $m \operatorname{dim} X_{r}$.

By Lemma 2.11 one may identify $\mathcal{A}_{1}^{\Delta} X$ with the $\Delta$-tangent bundle $T^{\Delta} X$ of $X$. Specializing Corollary 2.12 to the case of $m=1$ we see that for $a \in X$ sufficiently general, $K_{X}=K_{T_{a}^{\Delta} X}$. This fact is an old theorem of Kolchin [7].

\section{Dichotomy theorem}

Here we complete the promised proof that if $p$ is a non-locally modular regular type in a $\Delta$-closed field, then $p$ is nonorthogonal to a regular generic type of a definable subgroup of the additive group.

In what follows, we work inside a fixed universal $\Delta$-closed field $\mathbb{U}$. Both the statement of our main results as well as the methods depend heavily on the machinery of stability theory and its meaning in differential fields. The reader is referred to [13] and [11], but we recall some of the key notions.

We consider $\mathbb{U}$ as a structure in the language of rings together with $\partial_{1}, \ldots, \partial_{n}$. The first order theory of $\mathbb{U}$ (namely $\mathrm{DCF}_{0, n}$ ) is $\omega$-stable, so stable, and has quantifierelimination and elimination of imaginaries. Also $\mathbb{U}$ is a saturated model. Stability provides a notion of independence: $a \downarrow_{A} b$ (read as $\operatorname{tp}(a / A b)$ does not fork over $\left.A\right)$ where $a, b$ are tuples and $A$ is a set. In our context the meaning is: the differential fields generated by $A a$ and $A b$ are algebraically disjoint over the differential field generated by $A$. A complete type $p(x) \in S(A)$ is stationary if it has a unique nonforking extension over any $B$ containing $A$. In our context, any type over an algebraically closed differential field is stationary. We say that stationary types $p, q$ (over possibly different sets of parameters) are orthogonal, written $p \perp q$ if for any set $C$ of parameters containing $\operatorname{dom}(p)$ and $\operatorname{dom}(q)$, if $a$ and $b$ realize the nonforking extensions of $p, q$ respectively, to $C$, then $a \downarrow_{C} b$. The (stationary) type $p$ is said to be regular if it is orthogonal to all its forking extensions. If $p(x) \in S(A)$ is a regular type, its set of realizations forms a pregeometry with respect to forking over $A$. So for $a$ a tuple of realizations of $p, \operatorname{dim}(a)$ makes sense. The regular type $p(x) \in S(A)$ is said to be locally modular if (after possibly replacing $p(x)$ by a nonforking extension $p^{\prime}(x) \in S(B)$ ), the corresponding pregeometry is modular, meaning that for finite-dimensional closed sets of realizations of $p, X, Y$ say, $\operatorname{dim}(X)+\operatorname{dim}(Y)-\operatorname{dim}(X \cap Y)=\operatorname{dim}(X \cup Y)$. If $p(x)$ is the generic type of a definable field then $p$ is non locally modular. The optimal result in our context $\left(\mathrm{DCF}_{0, n}\right)$ would be that any non locally modular regular type is nonorthogonal to the (regular) generic type of a definable field (which would have to be a field of constants, possibly the whole field). This is the case for types of $\Delta$-type zero.

In addition to the afore-mentioned notions, we make use below of various other notions such as domination equivalence, $p$-weight, and semiregular types. Chapter 7 of [11] deals with this material.

If $p=\operatorname{tp}(a / A)$, by $m(p)$ we mean the $\Delta$-type of $a$ over the $\Delta$-field generated by the set $A$ (as defined at the end of the last section). Note that $m(p)=m\left(p^{\prime}\right)$ for $p^{\prime}$ a nonforking extension of $p$. If $X$ is an irreducible $\Delta$-variety, $m(X)$ denotes the $\Delta$-type of $X$. So $m(X)=m(p)$ where $p$ is the generic type of $X$. For a type $p, \operatorname{loc}(p)$ denotes the Kolchin closure of the set of realization of $p$. We often use $m(a / A)$ or $\operatorname{loc}(a / A)$ to mean $m(\operatorname{tp}(a / A))$ and $\operatorname{loc}(\operatorname{tp}(a / A))$, respectively. 
Lemma 3.1. The $\Delta$-type and typical $\Delta$-dimension are additive in the sense that - $m(a b)=\max \{m(a), m(b / a)\}$ - if $m(a)=m(b / a)$ then $\operatorname{dim}_{\Delta}(a b)=\operatorname{dim}_{\Delta}(a)+\operatorname{dim}_{\Delta}(b / a)$, and

- if $m(a)>m(b / a)$ then $\operatorname{dim}_{\Delta}(a b)=\operatorname{dim}_{\Delta}(a)$.

Proof. As the dimension functions are additive in fibrations, $K_{a b / F}(y)=K_{a / F}(y)+$ $K_{b / F a}(y)$. The lemma follows by computing the degree and leading coefficient of the sum of two polynomials.

We begin by investigating some relations between $\Delta$-type and regularity.

Definition 3.2. A regular type $p$ is $\Delta$-type minimal if for any other regular type $q, q \not \perp p \Rightarrow m(q) \geq m(p)$.

Question 3.3. Are there regular types in $\Delta$-closed fields which are not $\Delta$-type minimal?

We also consider a related, though distinct, minimality property for $\Delta$-varieties.

Definition 3.4. An irreducible $\Delta$-variety $X$ is $\Delta$-type minimal if $m(Y)<m(X)$ for any proper $\Delta$-subvariety $Y \subset X$.

Lemma 3.5. Let $r$ be a type and suppose that $X:=\operatorname{loc}(r)$ is $\Delta$-type minimal. Then $r$ is a regular type.

Proof. Our hypothesis implies already that $r$ is stationary as if $r$ were not stationary, then $X$ would have more than one component of $\Delta$-type $m(X)$.

Let $a$ realize a forking extension (to some algebraically closed $\Delta$-field $k$ ) of $r$ and $b$ realize the nonforking extension of $r$ to $k$. As $\operatorname{loc}(a b / k)$ maps dominantly to $X$ via the projection to the second coordinate, we see that $m(a b / k) \geq m(X)$. However, if $a \downarrow_{k} b$, then we would have $m(b / k, a)<m(X)$ and $m(a / k)<m(X)$ so that $m(a b / k)<m(X)$.

In the next lemma, we simply observe that the analysis behind the decomposition (up to domination equivalence) of a type as a product of regular types may be accomplished in such a way that the resulting regular types have $\Delta$-type no more than that of the original type.

Lemma 3.6. Let $q$ be any stationary type. Then there is a finite sequence of regular types $r_{1}, \ldots, r_{\ell}$ such that $m\left(r_{i}\right) \leq m(q)$ for all $i$ and $q$ is domination equivalent to $r_{1} \otimes \cdots \otimes r_{\ell}$.

Proof. It is enough to show that any regular type $r$ which is nonorthogonal to $q$ is nonorthogonal to some regular $r^{\prime}$ with $m\left(r^{\prime}\right) \leq m(q)$. Suppose $r$ is such. So (after passing to nonforking extensions over some $k$ ) there are realizations $a$ of $q$ and $b$ of $r$ such that $a \Downarrow_{k} b$. Let $c$ be the canonical base of $\operatorname{tp}(a / k, b)$. Then $c \notin a c l(k)$ and $c \in \operatorname{acl}(k, b)$. Thus $r^{\prime}=t p(c / k)$ is regular (as regularity is preserved by algebraicity) and nonorthogonal to $r$ (as $c \downarrow_{k} b$ ). On the other hand $c$ is contained in the definable closure of $k$ together with a finite sequence of realizations of $q$. Hence by Lemma 3.1, $m\left(r^{\prime}\right) \leq m(q)$.

Corollary 3.7. If $p$ is a $\Delta$-type minimal regular type, then for any (not necessarily regular) stationary type $r, r \not \perp p \Rightarrow m(r) \geq m(p)$. 
Proof. Suppose for a contradiction that $m(r)<m(p)$. Using Lemma 3.6 find regular types $r_{1}, \ldots, r_{\ell}$ with $m\left(r_{i}\right) \leq m(r)<m(p)$ for $i \leq \ell$ and $r \sqsubseteq r_{1} \otimes \cdots \otimes r_{\ell}$. As $p \not \perp r, p \not \perp r_{i}$ for some $i$; but this contradicts the $\Delta$-type minimality of $p$.

Definition 3.8. A $\Delta$-vector group is a group $H$ definable in $\mathbb{U}$ which is definably isomorphic to a subgroup of some Cartesian power of the additive group.

We note that the class of $\Delta$-vector groups is closed under taking definable subgroups and quotients. We also note that because every $\Delta$-vector group is a vector space over the field of $\Delta$-constants, every $\Delta$-vector group is divisible and therefore connected.

Lemma 3.9. Let $G$ be a $\Delta$-vector group. Then the $\Delta$-tangent space of $G$ at its origin is definably isomorphic to $G$. Moreover, if $H<G$ is a $\Delta$-subgroup, then the restriction to $H$ of the isomorphism between $G$ and $T_{0}^{\Delta} G$ is an isomorphism between $H$ and $T_{0}^{\Delta} H$.

Proof. Present $G$ as a $\Delta$-closed subgroup of $\mathbb{G}_{a}{ }^{g}$ for some $g$. For each $r \geq 0, \tau_{r}\left(\mathbb{G}_{a}{ }^{g}\right)$ is again some cartesian power of the additive group. Let $\phi_{r}: \tau_{r}\left(\mathbb{G}_{a}{ }^{g}\right) \rightarrow T \tau_{r}\left(\mathbb{G}_{a}{ }^{g}\right)$ be the map given by $x \mapsto(0, x)$ which identifies $\tau_{r}\left(\mathbb{G}_{a}{ }^{g}\right)$ with its tangent space at the origin, $T_{0} \tau_{r}\left(\mathbb{G}_{a}{ }^{g}\right)$. As an algebraic subgroup of $\tau_{r}\left(\mathbb{G}_{a}{ }^{g}\right)$, the defining ideal of $G_{r}:=\overline{\nabla_{r} G}$ is generated by (homogeneous) linear polynomials, and hence its tangent space at the origin is given by the same polynomials. That is, each $\phi_{r}$ restricts to an isomorphism from $G_{r}$ to $T_{0}\left(G_{r}\right)$. Recall that under the natural identifications $T_{0}\left(G_{r}\right)=\mathcal{A}_{1}\left(G_{r}\right)_{\nabla_{r}(0)}=\overline{\nabla_{r}\left(\mathcal{A}_{1}^{\Delta} G_{0}\right)}$ and $\mathcal{A}_{1}^{\Delta} G_{0}=T_{0}^{\Delta} G$. So $\left\langle\phi_{r} \mid r \geq 0\right\rangle$ identifies the prolongation sequence of $G$ with the prolongation sequence of $T_{0}^{\Delta} G$, and hence identifies $G$ with $T_{0}^{\Delta} G$ as desired. The "moreover" clause follows by our construction of the isomorphism.

Corollary 3.10. Suppose that $G$ is a $\Delta$-vector group such that $m(H)<m(G)$ for any proper definable subgroup $H<G$. Then $m(X)<m(G)$ for all proper $\Delta$-subvarieties $X \subset G$. In particular, the generic type of $G$ is regular.

Proof. Let $X \subseteq G$ be a $\Delta$-type minimal $\Delta$-subvariety of $G$ with $m(X)=m(G)$. After translating $X$ we may assume that $0 \in X$ is smooth. By Corollary 2.12 (which in this case is a theorem of Kolchin [7]), $m\left(T_{0}^{\Delta} X\right)=m(X)=m(G)$. Visibly, $T_{0}^{\Delta} X \leq T_{0}^{\Delta} G$, and by Lemma $3.9 T_{0}^{\Delta} G \cong G$. Thus, $T_{0}^{\Delta} X=T_{0}^{\Delta} G$. By Corollary 2.12 again, the Kolchin polynomial of $X$ and of $T_{0}^{\Delta} X$ agree (as do those of $G$ and $T_{0}^{\Delta} G$ ). Thus, $X=G$ as desired, by the comment preceding Corollary 2.12. By Lemma 3.5 the generic type of $G$ is regular.

We now analyze the relation between arc spaces and non local modularity. First we point out that the arcs give us information about canonical bases.

Lemma 3.11. Let $k$ be a small algebraically closed $\Delta$-field and $a$ and $c$ tuples. Let $X:=\operatorname{loc}(a / k)$. Suppose that $c=\operatorname{Cb}(a / k, c)$. Then there is an integer $m \geq 0$ and a tuple d from $\mathcal{A}_{m}^{\Delta} X_{a}$ with $c \in \operatorname{dcl}(k, a, d)$.

Proof. Let $Y:=\operatorname{loc}(a / k, c)$. We know that $c$ is interdefinable over $k$ with the canonical parameter for $Y$. By Lemma 2.10, the latter is interdefinable over $\operatorname{dcl}(k, a)$ with the sequence of canonical parameters of $\mathcal{A}_{m}^{\Delta} Y_{a}$ (considered as subsets of $\mathcal{A}_{m}^{\Delta} X_{a}$ ). By stability, each $\mathcal{A}_{m}^{\Delta} Y_{a}$ is definable with parameters from $\mathcal{A}_{m}^{\Delta} X_{a}$.

In general, arc spaces are not groups, but they are analyzable in terms of groups. 
Lemma 3.12. Let $X$ be $a \Delta$-variety over a $\Delta$-closed field $k$ and $a \in X$ a smooth point. Let $b \in \mathcal{A}_{m}^{\Delta} X_{a}$ be a point in an arc space of $X$ over a. Then there are elements $b_{1}, \ldots, b_{m}=b$, each in the definable closure of $k \cup\{a, b\}$ such that each $b_{i}$ is in some $k \cup\left\{a, b_{i-1}\right\}$-definable principal homogeneous space for $T_{a}^{\Delta} X$.

Proof. By (the proofs of) Lemmas 2.2 and 2.11 each fibre of $\rho_{i+1, i}: \mathcal{A}_{i+1}^{\Delta} X_{a} \rightarrow$ $\mathcal{A}_{i}^{\Delta} X_{a}$ is a principal homogeneous space for $T_{a}^{\Delta} X$. Set $b_{i}:=\rho_{m, i}(b)$.

Lemma 3.13. Let $k$ be $a \Delta$-closed field and $p \in S(k)$ a $\Delta$-type minimal regular type with $m(p)=m$. If $p$ is not locally modular, then there are a $\Delta$-vector group $G$ and a type $q$ having

- $m(q)=m(G)=m$,

- $q(x) \vdash x \in G$, and

- $p \not \perp q$

Proof. Suppose $p$ is not locally modular. We will make use of Corollary 5.2 from Chapter 8 of [11]. This says that, working over some algebraically closed set of parameters containing $k$, we can find $a \in p^{\mathrm{eq}}$ and $c \in p^{\mathrm{eq}}$ with $w_{p}(c / a)=1$, $w_{p}(a / c)=1, w_{p}(a)=2, c=\mathrm{Cb}(a / c)$, and such that $r:=\operatorname{tp}(c / a)$ is $p$-semiregular.

Note first that $m(a)=m$. This is because $a$ is in the definable closure of some finite tuple of realizations of $p$, and thus by Lemma 3.1, $m(a) \leq m$. But $t p(a)$ is nonorthogonal to $p\left(\right.$ as $\left.w_{p}(a)=2\right)$, hence by the choice of $p, m(a)=m$.

Now let $X=\operatorname{loc}(a)$, and let $G=T_{a}^{\Delta} X$. By Corollary 2.12. $m(G)=m$. We aim to show that $r$ is nonorthogonal to a type realized in $G$.

Claim I: $r$ is nonorthogonal to $\operatorname{tp}(e / A)$ for some set $A$ containing $a$ and some $e \in \mathcal{A}_{s}^{\Delta} X_{a}$ for some $s \geq 0$.

Proof of Claim I: By Lemma 3.11 there are some integer $s \geq 0$ and elements $e_{1}, \ldots, e_{\ell}$ of $\mathcal{A}_{s}^{\Delta} X_{a}$ so that $c \in \operatorname{dcl}\left(a, e_{1}, \ldots, e_{\ell}\right)$. Since $c \notin \operatorname{acl}(a)$, there is $i \leq l$ such that $c \downarrow_{a}\left(e_{1}, \ldots, e_{i-1}\right)$, but $c \Downarrow_{\left(a, e_{1}, \ldots, e_{i-1}\right)} e_{i}$. Put $A=\left\{a, e_{1}, \ldots, e_{i-1}\right\}$ and $e=e_{i}$, to yield the claim.

Claim II: $r$ is nonorthogonal to $t p(b / B)$ for some $B \supseteq A$ and $b \in T_{a}^{\Delta} X=G$.

Proof of Claim II: Let $b_{1}, \ldots, b_{t}=e$ be given by Lemma 3.12 applied to the $e \in \mathcal{A}_{m} X_{a}$ in Claim I. So for some $j, r$ is nonorthogonal to $\operatorname{tp}\left(b_{j} / A \cup\left\{b_{1}, \ldots, b_{j-1}\right\}\right)$. By Lemma $3.12 b_{j}$ is a member of an $\left\{a, b_{j-1}\right\}$-definable set which is in definable bijection with $G$. Hence clearly $r$ is nonorthogonal to a type realized in $G$, yielding Claim II.

As $w_{p}(c / a)=1$ and $r$ is $p$-semiregular, we actually have that $r$ is regular and nonorthogonal to $p$. So $p$ is nonorthogonal to a type $q$ realized in $G$. As $m(p)=$ $m(G)=m$, and $p$ is $\Delta$-type minimal, this type $q$ must also be of $\Delta$-type $m$. As we know $G$ to be a $\Delta$-vector group, the proof of the lemma is complete.

Lemma 3.14. Let $p$ be a $\Delta$-type minimal regular type. Suppose that there are $a \Delta$-vector group $G$ and a type $q$ such that $p \not \perp q, q(x) \vdash x \in G$, and $m(q)=$ $m(G)=m(p)$. Then there is a $\Delta$-vector group whose generic type is regular and nonorthogonal to $p$.

Proof. We work by induction on $\operatorname{ord}(G):=\left\langle m(G), \operatorname{dim}_{\Delta}(G), U(G)\right\rangle$.

Claim: We may assume that if $H<G$ is a proper definable subgroup of $G$, then $m(H)<m(G)$.

Proof of Claim: Suppose that $H<G$ and $m(H)=m(G)$. Let $\pi: G \rightarrow G / H$ be the natural quotient map. 
Let us first note that $\operatorname{ord}(G / H)<\operatorname{ord}(G)$ : Assume $G, H$ defined over $\emptyset$. Let $g$ be generic in $G$ over $\emptyset$. Then $g / H$ is a generic of $G / H$ over $\emptyset$. By elimination of imaginaries we may assume $g / H$ is a finite tuple from our universal domain, hence $m(g / H)$ and $\operatorname{dim}_{\Delta}(g / H)$ - which note are birational invariants - make sense. Note also that $m(g)=m(g, g / H)$ and likewise for $\operatorname{dim}_{\Delta}$. Finally, $\operatorname{dim}_{\Delta}(g /(g / H))=$ $\operatorname{dim}_{\Delta}(H) \neq 0$ (as $\left.H \neq 0\right)$. Hence, if $m(g)=m(g / H)$ then by Lemma 3.1(ii), $\operatorname{dim}_{\Delta}(g / H)<\operatorname{dim}_{\Delta}(g)$, showing that $\operatorname{ord}(G / H)<\operatorname{ord}(G)$.

Also $\operatorname{ord}(H)<\operatorname{ord}(G)$ since $\operatorname{dim}_{\Delta}(H) \leq \operatorname{dim}_{\Delta}(G)$ and $U(H)<U(G)$.

Replacing $q$ with a nonforking extension, we may assume that $H$ is definable over $\operatorname{dom}(q)$. Write $q=\operatorname{tp}(a / A)$. Set $\bar{q}:=\operatorname{tp}(\pi(a) / A)$ and $q^{\prime}:=\operatorname{tp}(a / A, \pi(a))$. Let $b \in a+H$ be independent from $a$ over $\{A, \pi(a)\}$. Set $q^{\prime \prime}:=\operatorname{tp}(a-b / A, b)$. Note that $q^{\prime \prime}$ is a translation of the nonforking extension of $q^{\prime}$ to $A \cup\{b\}$. Using transitivity, one sees that either $p \not \perp \bar{q}$ or $p \not \perp q^{\prime \prime}$. In either case, we conclude by induction. That is, if $p \not \perp \bar{q}$ then by Corollary $3.7 m(\bar{q}) \geq m(p)$. As $\bar{q}(x) \vdash x \in G / H$ we have $m(\bar{q}) \leq m(G / H) \leq m(G)=m(p)$. Thus, $m(\bar{q})=m(p)$ so that the hypotheses of this lemma apply with $\bar{q}$ in place of $q$ and $G / H$ in place of $G$. Likewise, in the case of $p \not \perp q^{\prime \prime}$ we may replace $q$ with $q^{\prime \prime}$ and $G$ with $H$.

Let $r$ be the generic type of $G$. By Corollary 3.10 and the above reduction, $r$ is regular. It remains to show that $p \not \subset r$. Taking nonforking extensions we may assume that $p \not \not^{a} q$. Again, we suppress the base parameters. Let $a=p$ and $b \models q$ with $a \Downarrow b$. As $r$ is the generic type of $G$, we can find $c_{1}, c_{2}$ realising $r$ such that $b \in \operatorname{dcl}\left(c_{1}, c_{2}\right)$. Hence $a \downarrow c_{1}, c_{2}$. Now suppose, for a contradiction, that $p \perp r$. Then $a \downarrow c_{1}$ and so $a \Downarrow c_{1} c_{2}$. As $c_{2} \models r$, our assumption that $p \perp r$ implies that $c_{2} \Downarrow c_{1}$. Hence $\operatorname{loc}\left(c_{2} / c_{1}\right)$ is a proper $\Delta$-subvariety of $G$ and so $m\left(c_{2} / c_{1}\right)<m$ by Corollary 3.10 and the reduction of Claim 1. As $m=m(G)=m(p)$, Corollary 3.7 implies that $p \perp \operatorname{tp}\left(c_{2} / c_{1}\right)$. But this contradicts $a \Downarrow_{c_{1}} c_{2}$. So $p \not \perp r$, as desired.

Corollary 3.15. Let $p$ be a regular non locally modular type. Then there is a $\Delta$-vector group $G$ whose generic type is regular and nonorthogonal to $p$.

Proof. Let $p^{\prime}$ be a regular type nonorthogonal to $p$, and of minimal $\Delta$-type with this property. By the transitivity of nonorthogonality for regular types, $p^{\prime}$ is $\Delta$-type minimal (in the sense of Definition 3.2). Lemma 3.13 tells us that $p^{\prime}$ satisfies the hypotheses of Lemma 3.14, which in turn tells us that there is a $\Delta$-vector group $G$ with a regular generic type $\mathfrak{g}_{G}$ such that $\mathfrak{g}_{G} \not \perp p^{\prime}$. Hence $p \not \perp \mathfrak{g}_{G}$.

Lemma 3.16. Let $G$ be a $\Delta$-vector group with regular generic type $\mathfrak{g}_{G}$. There is a definable subgroup of the additive group itself whose generic type is regular and nonorthogonal to $\mathfrak{g}_{G}$.

Proof. Realize $G$ as a definable subgroup of $\mathbb{G}_{a}{ }^{g}$ for some $g$. Now one of the $g$ projections of $G$ to $\mathbb{G}_{a}$, say $\pi$, must have infinite image. Let $a$ realize the generic type of $G$ (over the defining set, say $k$, of parameters). Then $\pi(a)$ realizes the generic type of $\pi(G)$. But $t p(\pi(a) / k)$ is also regular. Thus $H=\pi(G)$ is a definable subgroup of the additive group with generic type regular and nonorthogonal to the generic type of $G$.

Combining all the results of this section, we conclude with our main theorem.

Theorem 3.17. If $p$ is a regular non locally modular type, then there exists a definable subgroup of the additive group whose generic type is regular and nonorthogonal to $p$. 
Proof. By Corollary 3.15 there is some $\Delta$-vector group $G$ having a regular generic type $\mathfrak{g}_{G}$ nonorthogonal to $p$. By Lemma 3.16 there is a definable subgroup $H \leq$ $\mathbb{G}_{a}$ of the additive group having a regular generic $\mathfrak{g}_{H}$ nonorthogonal to $\mathfrak{g}_{G}$. As nonorthogonality is transitive for regular types, we have $p \not \perp \mathfrak{g}_{H}$.

\section{REFERENCES}

[1] S. Bosch, W. Lütkebohmert, and M. Raynaud, Néron models, Ergebnisse der Mathematik und ihrer Grenzgebiete (3), 21, Springer-Verlag, Berlin, 1990. x+325 pp.

[2] J. Denef and F. Loeser, Germs of arcs on singular algebraic varieties and motivic integration, Invent. Math. 135 (1999), no. 1, 201-232.

[3] D. Haskell, E. Hrushovski, and D. Macpherson, Definable sets in algebraically closed valued fields: elimination of imaginaries, Journal für die reine und angewandte Mathematik 597 (2006), 175-236.

[4] E. Hrushovski, The Mordell-Lang conjecture for function fields, J. Amer. Math. Soc. 9 (1996), no. 3, 667-690.

[5] E. Hrushovski and Ž. Sokolović, Minimal types in differentially closed fields, preprint 1992.

[6] E. Hrushovski and B. Zilber, Zariski Geometries, Journal of the American Mathematical Society, 9 (1996), 1-56.

[7] E. Kolchin, Differential algebraic groups, Pure and Applied Mathematics, 114, Academic Press, Inc., Orlando, FL, 1985, xvii+271 pp.

[8] E. Looijenga, Motivic measures, Séminaire Bourbaki, Vol. 1999/2000, Astérisque No. 276, (2002), 267-297.

[9] T. MCGrail, The model theory of differential fields with finitely many commuting derivations, J. Symbolic Logic 65 (2000), no. 2, 885-913.

[10] J. NAsh, Arc structure of singularities, A celebration of John F. Nash, Jr, Duke Math. J. 81 (1995), no. 1, 31-38.

[11] A. PIllay, Geometric Stability Theory, Oxford University Press, 1996.

[12] A. Pillay and M. Ziegler, Jet spaces of varieties over differential and difference fields, Selecta Math. New ser. 9 (2003), 579-599.

[13] B. Poizat, A Course in Model Theory: An introduction to contemporary mathematical logic, Translated from the French by Moses Klein and revised by the author, Universitext. Springer-Verlag, New York, 2000. xxxii+443 pp.

[14] A. Robinson, Complete Theories, North-Holland, Amsterdam, 1956.

[15] W. Sit, Well-ordering of certain numerical polynomials, Transactions of the American Mathematical Society, 212 (1975), 37-45.

[16] Y. Yaffe, Model completion of Lie differential fields, Ann. Pure Appl. Logic 107 (2001), no. $1-3,49-86$.

E-mail address: rmoosa@math.uwaterloo.ca

University of Waterloo, Department of Pure Mathematics, 200 University Avenue West, Waterloo, Ontario, N2L 3G1, Canada

E-mail address: pillay@math.uiuc.edu

University of Illinois at Urbana-Champaign, Department of Mathematics, Altgeld Hall, 1409 W. Green St., Urbana, IL 61801, USA

University of Leeds, School of Mathematics, Leeds LS2 9JT, England

E-mail address: scanlon@math.berkeley.edu

University of California, Berkeley, Department of Mathematics, Evans Hall, BerkeLEY, CA 94720-3480, USA 